TITLE:

\title{
Glass transitions and dynamics in thin polymer films: Dielectric relaxation of thin films of polystyrene
}

\author{
$\operatorname{AUTHOR}(\mathrm{S}):$
}

Fukao, K; Miyamoto, Y

\section{CITATION:}

Fukao, K ... [et al]. Glass transitions and dynamics in thin polymer films: Dielectric relaxation of thin films of polystyrene. Physical Review E 2000, 61(2): 1743-1754

ISSUE DATE:

2000-02

URL:

http://hdl.handle.net/2433/50237

RIGHT:

Copyright 2000 American Physical Society 


\title{
Glass transitions and dynamics in thin polymer films: Dielectric relaxation of thin films of polystyrene
}

\author{
K. Fukao* and Y. Miyamoto \\ Faculty of Integrated Human Studies, Kyoto University, Kyoto 606-8501, Japan
}

(Received 8 July 1999)

\begin{abstract}
The glass transition temperature $T_{g}$ and the temperature $T_{\alpha}$ corresponding to the peak in the dielectric loss due to the $\alpha$ process have been simultaneously determined as functions of film thickness $d$ through dielectric measurements for polystyrene thin films supported on glass substrate. The dielectric loss peaks have also been investigated as functions of frequency for a given temperature. A decrease in $T_{g}$ was observed with decreasing film thickness, while $T_{\alpha}$ was found to remain almost constant for $d>d_{\mathrm{c}}$ and to decrease drastically with decreasing $d$ for $d<d_{\mathrm{c}}$. Here, $d_{c}$ is a critical thickness dependent on molecular weight. The relaxation time $\tau_{\alpha}$ of the $\alpha$ process, which was measured as the frequency at which the dielectric loss realizes its peak value at a given temperature, was found to have a $d$ dependence similar to that of $T_{\alpha}$. The relaxation function for the $\alpha$ process was obtained by using the observed frequency dependence of the peak profile of the dielectric loss. The exponent $\beta_{\mathrm{KWw}}$, which was obtained from the relaxation functions, decreases as thickness decreases. This suggests that the distribution of relaxation times for the $\alpha$ process broadens with decreasing thickness. The thickness dependence of $T_{g}$ is directly related to the distribution of relaxation times for the $\alpha$ process, not to the relaxation time itself. The value of the thermal expansion coefficient normal to the film surface was found to increase with decreasing film thickness below $T_{g}$, but to decrease with decreasing film thickness above $T_{g}$. These experimental results are discussed in the context of a three-layer model in which within thin films there are three layers with different mobilities and glass transition temperatures.
\end{abstract}

PACS number(s): 64.70.Pf, 68.60.-p, 77.22.Gm

\section{INTRODUCTION}

Recently, glass transitions in amorphous materials have been investigated by many researchers. However, the mechanism involved in glass transitions is not yet fully understood [1-3]. Understanding the behavior of the characteristic length scale of the dynamics of supercooled liquids near the glass transition is the most important problem to be solved in such studies. In the Adam and Gibbs's theory, it is assumed that there is a domain in which collective particle motion can occur and its size grows as the temperature is lowered. This domain is called the cooperatively rearranging region (CRR) [4]. In connection with the CRR, recent molecular dynamics simulations have revealed the existence of significant large-scale heterogeneity in particle displacements, socalled dynamical heterogeneity in supercooled liquids [5-8]. As the temperature decreases toward $T_{g}$, the dynamical heterogeneity grows. Experimental studies using multidimensional NMR [9], dielectric hole burning [10], and photobleaching [11] have produced evidence of dynamical heterogeneity. These topics concerning heterogeneity are closely related to the length scale of dynamics near glass transitions.

Glass transitions in finite systems confined to nanopores [12-14] and thin films [15-22] have recently attracted much attention, because such systems can be regarded as model systems for studying the length scale of glass transitions. In such systems deviation from bulk properties is expected to

\footnotetext{
*Author to whom correspondence should be addressed. Electronic address: fukao@phys.h.kyoto-u.ac.jp
}

appear if the system size is comparable to the characteristic length scale. In particular, $T_{g}$ and the thermal expansion coefficient $\alpha_{\mathrm{n}}$ of thin films have been measured using several experimental techniques, including ellipsometry $[15,16]$, positron annihilation lifetime spectroscopy (PALS) [17], Brillouin light scattering [18,19], and x-ray reflectivity [20]. For the first time, Keddie et al. investigated $T_{g}$ and the thermal expansion coefficient $\alpha_{n}$ of thin polymer films supported on substrate. For polystyrene films on hydrogen-passivated $\mathrm{Si}, T_{g}$ was found to decrease with decreasing film thickness $d$ for $d<40 \mathrm{~nm}$ [15]. The value of $\alpha_{n}$ below $T_{g}$ was found to increase with decreasing $d$ approaching the value characterizing liquid states. It was suggested that this decrease in $T_{g}$ is caused by the presence of a liquidlike layer at the polymerair interface in this case; in the case of freely standing polystyrene films, $T_{g}$ decreases much more rapidly with decreasing film thickness [18]. These results suggest that the interaction between polymers and the substrate competes with surface effects. This competition leads to a more gradual decrease of $T_{g}$ in the former case. For a strong attractive interaction between polymers and the substrate, an increase in $T_{g}$ with decreasing $d$ was observed [16].

Positron annihilation lifetime measurements reveal that the observed $T_{g}$ values of supported PS films are similar to those obtained by Keddie et al. However, the thermal expansion coefficient obtained by PALS is independent of $d$ below $T_{g}$, while it decreases with decreasing thickness above $T_{g}$. It was proposed that there is a dead layer near the interface between polymers and the substrate in addition to a liquidlike layer at the polymer-air interface. In the case of thin polymer films supported on substrate, the glass transition temperature and thermal properties strongly depend on the 
competetion between interfacial and surface effects. There are still controversial experimental results for such systems.

The dynamics related to the glass transition in thin films have been investigated using several methods [23-25]. Second harmonic generation reveals that the distribution of relaxation times broadens with decreasing film thickness, while the average relaxation time of the $\alpha$ process remains constant for supported films of a random copolymer [23]. Ultrasonic measurements [24] have shown that the temperature $T_{\max }$ at which the ultrasonic absorption exhibits a maximum for a given frequency has a $d$ dependence similar to that of $T_{\mathrm{g}}$ obtained by ellipsometric measurements for thin polystyrene films supported on substrate. In the case of freely standing films of polystyrene, photon correlation spectroscopy [24] studies indicate that the relaxation behavior of the $\alpha$ process in thin films is similar to that of bulk samples of polystyrene, except for the reduction of the $\alpha$ relaxtion time. Atomic force microscopy studies [25] have revealed the existence of a mobile layer near the free surface of films of polystyrene. Because there are only a few experimental observations on the dynamics of thin polymer films, it is not yet clear whether properties of the $\alpha$ process change together with $T_{\mathrm{g}}$ as the film thickness decreases or whether the obtained results depend on the methods used for measurements or on the details of the individual samples.

Dielectric measurement is one of the most powerful experimental tools to investigate the dynamics of the $\alpha$ process in amorphous materials. Recently we applied this method to the determination of the glass transition temperature through measurements of the thermal expansion coefficient [26]. Bauer et al. also used this method and further extended it to thermal expansion spectroscopy $[27,28]$. By virture of dielectric measurements, it is possible to simultaneously measure the glass transition temperatures and determine the relaxation behavior of the $\alpha$ process of a single sample even for thin films.

In a previous paper [26], we reported that $T_{g}$ for thin polystyrene films supported on glass substrate can be determined from the temperature change of the electric capacitance during heating and cooling processes and that the dynamics of the $\alpha$ process can be determined from the dielectric loss of the films. We were able to obtain the distinct thickness dependences of $T_{\mathrm{g}}$ and $T_{\alpha}$ in which the dielectric loss exhibits a peak value for a fixed frequency due to the $\alpha$ process. In this paper the results obtained through dielectric measurements are described in detail, and the dynamics of the $\alpha$ process are investigated by measurements of the frequecncy dispersion of the dielectirc loss for the purpose of clarifying the relation involving the thickness dependence of $T_{g}$, the thermal expasion coefficients, and the dynamics of the $\alpha$ process. Based on the results of simultaneous measurements, we discuss the relationship between $T_{g}$ and the dynamics of the $\alpha$ process of thin polymer films. A possible explanation of our experimental results for $T_{g}(d)$ and the dynamics of the $\alpha$ process is given in terms of a three-layer model. This paper consists of five sections. In Sec. II experimental details and principles regarding our determination of $T_{g}$ and the thermal expansion coefficient using electric capacitance measurements are given. The experimental results for the thermal expansion coefficient $\alpha_{\mathrm{n}}$ and $T_{g}$ obtained from our measurements are given in Sec. III. A three-layer model, which can account for the observed thickness dependences of $\alpha_{\mathrm{n}}$ and $T_{g}$, is also introduced there. In Sec. IV the dynamics of the $\alpha$ process of thin films is investigated in reference to the peak profile in the dielectirc loss due to the $\alpha$ process in the frequency domain. In Sec. V overall discussion and a summary of this paper are given.

\section{EXPERIMENTAL DETAILS}

\section{A. Sample preparation and measurement procedures}

Four different atactic polystyrenes ( $a$-PS) were used. These were purchased from Scientific Polymer Products, Inc. $\left(M_{w}=2.8 \times 10^{5}\right)$, the Aldrich Co., Ltd. $\left(M_{w}=1.8 \times 10^{6}\right.$, $\left.M_{w} / M_{n}=1.03\right)$, and Polymer Source, Inc. $\left(M_{w}=3.6 \times 10^{4}\right.$, $M_{w} / M_{n}=1.06$, and $\left.M_{w}=3.6 \times 10^{3}, M_{w} / M_{n}=1.06\right)$. Thin films of $a$-PS with various thicknesses from 6 to $489 \mathrm{~nm}$ were prepared on an Al-deposited slide glass using a spincoat method from a toluene solution of $a$-PS. The thickness was controlled by changing the concentration of the solution. After annealing at $70{ }^{\circ} \mathrm{C}$ in the vacuum system for several days to remove solvents, Al was vacuum-deposited again to serve as an upper electrode. Heating cycles in which the temperature was changed between room temperature and $110^{\circ} \mathrm{C}\left(>T_{\mathrm{g}}\right)$ were applied prior to the dielectric measurements to relax the as-spun films and obtain reproducible results. "Bulk' films of $a$-PS (each with $d>100 \mu \mathrm{m}$ ) were made by oil-pressing samples melted at about $200{ }^{\circ} \mathrm{C}$ for a few minutes, and gold was vacuum-deposited onto both sides of the films to serve as electrodes. Dielectric measurements were done using an impedance analyzer (HP4284A) in the frequency range from $20 \mathrm{~Hz}$ to $1 \mathrm{MHz}$ during heating (cooling) processes in which the temperature was changed at a rate of $2 \mathrm{~K} / \mathrm{min}$. For dielectric measurements of very thin films, the resistance of the electrodes cannot be neglected. This leads to an extra loss peak on the high frequency side, which results from the fact that the system is equivalent to a series circuit of a capacitor and resistor, where the capacitance is that of the sample and the resistance is that of the electrodes [29]. The peak shape in the frequency domain can be fitted well by a simple Debye-type equation. Data obtained in the frequency domain, therefore, can be accurately corrected by subtracting the " $C-R$ peak' and assuming the validity of the Debye equation. Data corrected in this manner were used for further analysis in this paper.

\section{B. Relation involving the electric capacitance, thickness, and thermal expansion coefficients}

In this section we give the relation between the temperature change of the electric capacitance and thermal expansion coefficient. Similar discussion was also given by Bauer et al. [27]. In our measurements film thickness was evaluated from the capacitance at room temperature of as-prepared films by using the formula for the capacitance $C^{\prime}$ of a flat-plate condenser, $C^{\prime}=\epsilon^{\prime} \epsilon_{0} S / d$, where $\epsilon^{\prime}$ is the permittivity of $a$-PS, $\epsilon_{0}$ is the permittivity of the vacuum, $S$ is the area of the electrode $\left(S=8.0 \mathrm{~mm}^{2}\right)$, and $d$ is the thickness of the films. In general, the geometrical capacitance is given by

$$
C_{0}(T) \equiv \epsilon_{0} \frac{S}{d} \sim \epsilon_{0} \frac{S_{0}}{d_{0}}\left[1+\left(2 \alpha_{t}-\alpha_{n}\right) \Delta T\right],
$$




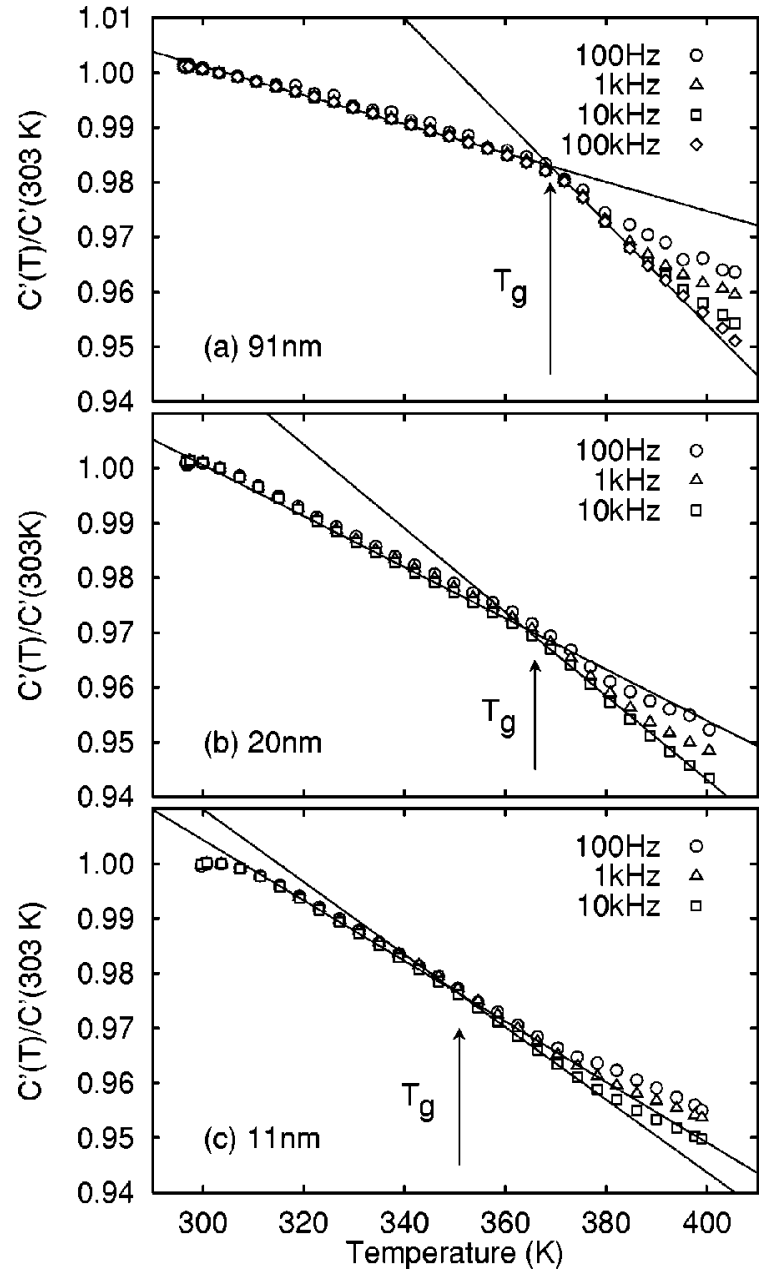

FIG. 1. Temperature dependence of the capacitance normalized with respect to the values at $303 \mathrm{~K}$ during the heating process for various frequencies from $100 \mathrm{~Hz}$ to $10 \mathrm{kHz}$ and three different thicknesses: (a) $d=91 \mathrm{~nm}$ and $M_{w}=1.8 \times 10^{6}$; (b) $d=20 \mathrm{~nm}$ and $M_{w}=2.8 \times 10^{5}$; (c) $d=11 \mathrm{~nm}$ and $M_{w}=1.8 \times 10^{6}$. The solid lines were obtained by fitting the data for $10 \mathrm{kHz}$ to a linear function below and above $T_{g}$. The arrows indicate the values of $T_{g}$.

and the permittivity is expressed by

$$
\epsilon^{\prime}(\omega, T)=\epsilon_{\infty}(T)+\epsilon_{\mathrm{disp}}(\omega, T),
$$

where $\epsilon_{\infty}$ is the permittivity in the high-frequency limit, $\alpha_{t}$ is the linear thermal expansion coefficient parallel to the film surface, $\alpha_{n}$ is the linear thermal expansion coefficient normal to the film surface, $\Delta T=T-T_{0}$, and $T_{0}$ is a standard temperature. The second term $\epsilon_{\text {disp }}$ on the right-hand side of Eq. (2) is related to the frequency dispersion of the dielectric loss due to the $\alpha$ process, the $\beta$ process, and so on.

If we here assume that the films are constrained along the substrate surface, the values of $\alpha_{t}$ and $\alpha_{n}$ are given by

$$
\alpha_{t}=0 \quad \text { and } \quad \alpha_{n}=\frac{1+\nu}{1-\nu} \alpha_{\infty},
$$

where $\nu$ is Poisson's ratio and $\alpha_{\infty}$ is the bulk linear coefficient of thermal expansion [20]. It should be noted that this case corresponds to that of "constant area conditions" in Ref. [27].
In the temperature range where the effect of the dielectric dispersion, i.e., the second term in the expression for $\epsilon^{\prime}$, can be neglected, we obtain

$$
\epsilon^{\prime}(T)=\epsilon_{\infty}(T) \sim \epsilon_{\infty}\left(T_{0}\right)\left(1-\eta_{0} \alpha_{n} \Delta T\right),
$$

where

$$
\begin{gathered}
\boldsymbol{\epsilon}_{\infty}\left(T_{0}\right)=\frac{1+2 \xi_{0}}{1-\xi_{0}}, \\
\eta_{0} \equiv \frac{3 \xi_{0}}{\left(1-\xi_{0}\right)\left(1+2 \xi_{0}\right)}, \\
\xi_{0} \equiv \frac{1}{3 \epsilon_{0}} \sum_{j} N_{j, 0} \bar{\alpha}_{j} .
\end{gathered}
$$

Here $N_{j, 0} \equiv N_{j}\left(T_{0}\right), N_{j}(T)$ is the number density of the $j$ th atom at $T$, and $\bar{\alpha}_{j}$ is the polarizability of the $j$ th atom. The Clausius-Mossotti relation, $\left(\epsilon_{\infty}-1\right) /\left(\epsilon_{\infty}+2\right)$ $=1 / 3 \epsilon_{0} \cdot \Sigma N_{j} \bar{\alpha}_{j}$, where $N_{j}(T)=N_{j}\left(T_{0}\right)\left(1-\alpha_{n} \Delta T\right)$, has been used.

In the case of $a$-PS, the dielectric constant $\epsilon^{\prime}$ of bulk samples is 2.8 at room temperature [30]. If we assume that $\epsilon^{\prime}\left(T_{0}\right) \approx \epsilon_{\infty}\left(T_{0}\right)=2.8$, then $\xi_{0}=0.375$ and $\eta_{0}$ is nearly equal to 1 . Using Eqs. (1), (3), and (4), we obtain the temperature coefficient of the capacitance $\tilde{\alpha}$ as follows:

$$
\begin{aligned}
\tilde{\alpha} & \equiv-\frac{1}{C^{\prime}\left(T_{0}\right)} \frac{d C^{\prime}(T)}{d T}=-\left(\frac{1}{\epsilon^{\prime}\left(T_{0}\right)} \frac{d \epsilon^{\prime}}{d T}+\frac{1}{C_{0}\left(T_{0}\right)} \frac{d C_{0}}{d T}\right) \\
& =\left(1+\eta_{0}\right) \alpha_{n} \approx 2 \alpha_{n} .
\end{aligned}
$$

We thus see that the temperature coefficient of $C^{\prime}$ is proportional to $\alpha_{n}$. It is therefore expected that the temperature coefficient changes at $T_{g}$. In the literature [31] we find $\nu$ $=0.325$ and $\alpha_{\infty}=0.57 \times 10^{-4} / \mathrm{K}$ for $T<T_{g}$, and $\nu=0.5$ and $\alpha_{\infty}=1.7 \times 10^{-4} / \mathrm{K}$ for $T>T_{g}$. Hence, for bulk samples of $a$-PS, it can be expected that

$$
\tilde{\alpha}= \begin{cases}2.2 \times 10^{-4}, & T<T_{g} \\ 10.2 \times 10^{-4}, & T>T_{g} .\end{cases}
$$

\section{GLASS TRANSITION TEMPERATURE OF THIN FILMS}

\section{A. Glass transition temperature and thermal expansion coefficients}

Figure 1 displays the temperature dependence of the capacitance, normalized with respect to the value at $303 \mathrm{~K}$ during the heating processes. In Fig. 1(a) we can see that the values at thickness $91 \mathrm{~nm}$ for different frequencies fall along a single line and decrease with increasing temperature for the temperature range from room temperature to approximately $370 \mathrm{~K}$. At higher temperature the normalized capacitance decreases with increasing temperature more steeply than at lower temperature. In this range the values for different frequencies can no longer be fitted by a single line. Here, they are dispersed due to the appearance of the $\alpha$ process. For the temperature range shown in the figure, however, it is appar- 


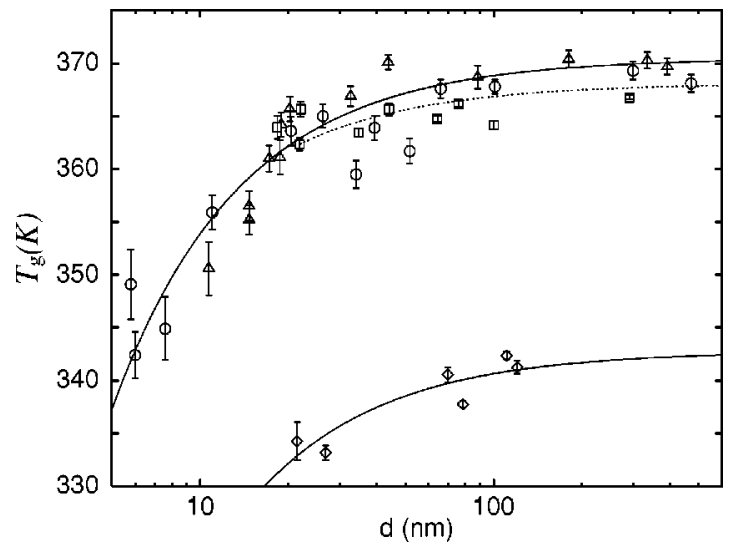

FIG. 2. Thickness dependence of $T_{g}$ of $a$-PS films obtained during the heating process ( $\triangle$ corresponds to $M_{w}=1.8 \times 10^{6}, \bigcirc$ to $M_{w}=2.8 \times 10^{5}, \square$ to $M_{w}=3.6 \times 10^{4}$, and $\diamond$ to $\left.M_{w}=3.6 \times 10^{3}\right)$. The values of $T_{g}$ are determined as the crossover temperatures between the lines characterizing $C^{\prime}(T)$ at $10 \mathrm{kHz}$ below and above $T_{g}$. The curves were obtained from the equation $T_{g}(d)=T_{g}^{\infty}(1$ $-a / d)$. The upper solid curve is for $M_{w}=1.8 \times 10^{6}$ and $2.8 \times 10^{5}$, the lower solid curve is for $M_{w}=3.6 \times 10^{3}$, and the dotted curve is for $M_{w}=3.6 \times 10^{4}$.

ent that the effect of the dispersion is quite weak above 10 $\mathrm{kHz}$. Therefore, for such frequencies the temperature at which the slope of $C^{\prime}(T)$ changes discontinuously can be determined unambiguously as the crossover temperature between the line characterizing the lower temperature side and that characterizing the higher temperature side. This crossover temperature can be regarded as $T_{g}$, because the thermal expansion coefficient changes through the crossover temperature, as can be expected from the discussion in Sec. II. As $d$ decreases, $T_{g}$ also decreases, as shown in Figs. 1(b) and 1(c).

Figure 2 displays the $d$ dependence of $T_{g}$ for $a$-PS films with four different molecular weights. In each case, the values of $T_{g}$ for the various values of $d$ were determined as the crossover temperatures at which the temperature coefficient of the capacitance at $10 \mathrm{kHz}$ changes during the heating process. For thick films, the values of $T_{g}$ are almost equal to those for bulk PS. When the films are thinner than about 100 nm, however, a decrease in $T_{g}$ is observed. The value of $T_{g}$ for films of $6 \mathrm{~nm}$ thickness is lower by about $30 \mathrm{~K}$ than that of films of $489 \mathrm{~nm}$ thickness for $a$-PS films with $M_{w}=2.8$ $\times 10^{5}$. The dependence of $T_{g}$ on $d$ can be expressed as

$$
T_{g}(d)=T_{g}^{\infty}\left(1-\frac{a}{d}\right),
$$

where $T_{g}(d)$ is the measured glass transition temperature for a film of thickness $d$. The values of the parameters resulting in the best fit are listed in Table I.

The asymptotic value $T_{g}^{\infty}$ has a distinct molecular weight dependence. For bulk samples of $a$-PS, the variation in $T_{g}$ with molecular weight is described well by the empirical equation

$$
T_{g}^{\infty}=\widetilde{T}_{g}^{\infty}-\frac{C}{N},
$$

TABLE I. The values of the parameters resulting in the best fit of the glass transition temperatures $T_{g}(d)$ to Eq. (10) for thin films of $a$-PS with various molecular weights $\left(M_{w}=3.6 \times 10^{3}-1.8\right.$ $\left.\times 10^{6}\right)$.

\begin{tabular}{lcc}
\hline \hline$M_{w}$ & $a(\mathrm{~nm})$ & $T_{g}^{\infty}(\mathrm{K})$ \\
\hline $1.8 \times 10^{6}, 2.8 \times 10^{5}$ & $0.45 \pm 0.06$ & $370.5 \pm 0.9$ \\
$3.6 \times 10^{4}$ & $0.33 \pm 0.05$ & $367.8 \pm 0.5$ \\
$3.6 \times 10^{3}$ & $0.62 \pm 0.12$ & $342.8 \pm 1.1$ \\
\hline \hline
\end{tabular}

where $N$ is the degree of polymerization, $\widetilde{T}_{g}^{\infty}=373 \mathrm{~K}$, and $C=1.1 \times 10^{3}$ [33]. Using Eq. (11), we obtain the values of $T_{g}^{\infty}$ as follows: $T_{g}^{\infty}=373 \mathrm{~K}$ for $M_{w}=1.8 \times 10^{6}$ and $2.8 \times 10^{5}$, $370 \mathrm{~K}$ for $M_{w}=3.6 \times 10^{4}$, and $341 \mathrm{~K}$ for $M_{w}=3.6 \times 10^{3}$. Therefore, it is found that the $M_{w}$ dependence of $T_{g}$ for the bulk samples can be reproduced quite well by the present measurements.

As shown in Table I, the length scale $a$ related to the thickness dependence of $T_{\mathrm{g}}$ ranges from $0.3-0.6 \mathrm{~nm}$. Taking into account the scatter in $a$, however, there is no distinct molecular weight dependence of $a$. This length scale is of the same order as that of the statistical segment of polystyrene $(0.68 \mathrm{~nm})$ [32]. These experimental results for $T_{g}^{\infty}$ and $a$ suggest that the thickness dependence of $T_{g}$ is almost independent of the molecular weight of $a$-PS after rescaling with respect to $T_{g}$ of bulk samples. This seems to be consistent with the fact that the length scale $a$ is not related to chain lengths but rather to segment lengths.

If our data are fitted by the function proposed by Keddie et al., $T_{g}(d)=T_{g}^{\infty}\left[1-(a / d)^{\tilde{\delta}}\right]$, the observed data for $M_{w}$ $=1.8 \times 10^{6}$ and $2.8 \times 10^{5}$ can also be fitted to this equation, and the parameter values resulting in the best fit are as follows: $a=0.39 \pm 0.10 \mathrm{~nm}$ and $\widetilde{\delta}=0.96 \pm 0.08$. The revised value of $\widetilde{\delta}$ obtained by Keddie and Jones is $\widetilde{\delta}=1.28 \pm 0.20$ [34]. This suggests that Keddie's equation can be replaced by Eq. (10) ( $\widetilde{\delta} \approx 1)$. The values $T_{g}$ obtained in our measurements also agree well with those obtained by Forrest et al. [19]. Thus, it can be concluded that $T_{g}$ of thin films has been accurately determined by measurements of the electric capacitance. With the present method it has been confirmed that the apparent $T_{g}$ obtained in our measurements decreases with decreasing film thickness.

Since no general theory for the effect on the glass transition of finite size has yet been presented, Eq. (10) is just an experimental findings at the present stage. As discussed later, in the context of the three-layer model we regard the glass transition temperature described by Eq. (10) as that associated with the $\alpha$ process (in this case, segmental motions of polymer chains) in the bulklike layer between the dead layer and the liquidlike one. The observed decrease in $T_{g}$ may be attributed to the distribution of the relaxation times of the $\alpha$ process, which comes from the existence of a boundary between the liquidlike layer and the bulklike layer, and also between the bulklike layer and dead layer.

Here, it should be noted that the temperature coefficient $\tilde{\alpha}$ of electric capacitance also changes with film thickness, as shown in Fig. 1. To clarify the $d$ dependence of $\tilde{\alpha}$, Fig. 3 displays $\tilde{\alpha}$ as a function of the inverse of the film thickness 


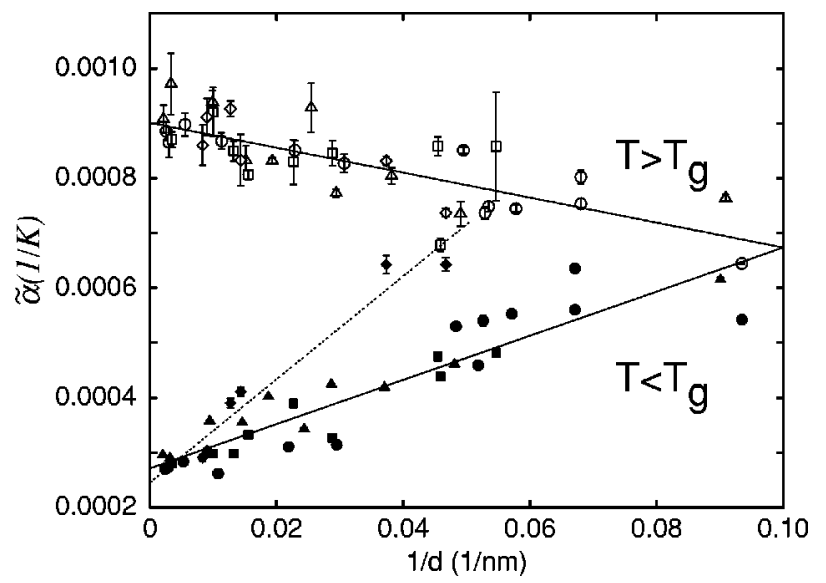

FIG. 3. Thickness dependence of the temperature coefficient of the capacitance of $a$-PS films obtained by measurements of the electric capacitance at $10 \mathrm{kHz}$ during the heating process. Above $T_{g}$, the symbol $\bigcirc$ correspond to $M_{w}=1.8 \times 10^{6}, \triangle$ to $M_{w}=2.8$ $\times 10^{5}, \square$ to $M_{w}=3.6 \times 10^{4}$, and $\diamond$ to $M_{w}=3.6 \times 10^{3}$. Below $T_{g}$, the filled symbols are used, and the correspondence between the symbols and the molecular weights is the same as above $T_{g}$. The lines were obtained by fitting the data to Eqs. (12) and (13). The dotted line is valid only for the data of the films with $M_{w}=3.6$ $\times 10^{3}$ below $T_{g}$.

for thin films. It is found that $\tilde{\alpha}$ increases with decreasing thickness below $T_{g}$, while it decreases with decreasing film thickness above $T_{g}$. In both cases, the thickness dependence of $\tilde{\alpha}$ can be expressed as a linear function of the inverse of film thickness. This observed $d$ dependence of $\tilde{\alpha}$ seems to be independent of the molecular weight within experimental accuracy, except for the case with $M_{w}=3.6 \times 10^{3}$ below $T_{g}$. The values of $\tilde{\alpha}$ for bulk samples can be obtained by taking 1/d to zero as follows: $\tilde{\alpha}=9.0 \times 10^{-4} \mathrm{~K}^{-1}$ for $T>T_{g}$ and $2.8 \times 10^{-4} \mathrm{~K}^{-1}$ for $T<T_{g}$. These values agree well with those predicted by Eq. (9) in Sec. II, and hence the temperature coefficient of the electric capacitance $\tilde{\alpha}$ observed in the present measurements can be regarded as the linear thermal expansion coefficient normal to the substrate $\alpha_{\mathrm{n}}$ multiplied by a factor of 2, as shown in Eq. (8). This $d$ dependence of $\tilde{\alpha}$ and $\alpha_{\mathrm{n}}$ can be attributed to the existence of some layers with different chain mobilities within thin polymer films supported on substrate, as discussed in the next section.

\section{B. Three-layer model}

In order to explain the observed thickness dependence of $\tilde{\alpha}$, we introduce a three-layer model, in which it is assumed that a thin polymer film on substrate consists of three layers with different molecular mobilities [17]. Near the interface between the glass substrate and polymers there is a dead layer which has almost no mobility. On the other hand, near the free surface there is a liquidlike layer which has higher mobility. Here, we assume that the thicknesses of the two layers are $\delta$ (dead layer) and $\xi$ (liquidlike layer). Between these two layers there is a bulklike layer which has the same mobility as that of the bulk samples. In this model, below the apparent $T_{g}$ the observed linear thermal expansion coefficient $\alpha_{n}$ normal to the surface of the substrate is given by

$$
\alpha_{n}=\frac{\xi}{d} \alpha_{1}^{\infty}+\left(1-\frac{\delta+\xi}{d}\right) \alpha_{g}^{\infty},
$$

and above $T_{g}$ by

$$
\alpha_{n}=\left(1-\frac{\delta}{d}\right) \alpha_{1}^{\infty}
$$

where $\alpha_{g}^{\infty}\left(\alpha_{1}^{\infty}\right)$ is the linear thermal expansion coefficient of the bulk glassy (liquid) state. Therefore, this simplified model can reproduce the observed thickness dependence of $\widetilde{\alpha}\left(=2 \alpha_{\mathrm{n}}\right)$ both below and above $T_{g}$. By fitting the observed results given in Fig. 3 to Eqs. (12) and (13), the thicknesses of dead and liquidlike layers are obtained: $\delta=(2.5$ $\pm 0.3) \mathrm{nm}$ and $\xi=(7.5 \pm 0.3) \mathrm{nm}$. Keddie et al. estimated the thickness of a liquidlike layer near the free surface to be $(8.0 \pm 0.8) \mathrm{nm}$. DeMaggio et al. obtained the thickness of the dead layer between polymer films and the substrate to be $(5.0 \pm 0.5) \mathrm{nm}$ and also proposed the existence of a mobile surface layer with thickness $2 \mathrm{~nm}$. The values of $\delta$ and $\xi$ observed in the present dielectric measurements are found to be consistent with those obtained by other experimental techniques: PALS and ellipsometry. Therefore, we conclude that the three-layer model can successfully be applied in our case.

The values of $\delta$ and $\xi$ for the lowest molecular weight sample $\left(M_{w}=3.6 \times 10^{3}\right)$ are $\delta=(3.7 \pm 1.0) \mathrm{nm}$ and $\xi$ $=(15.2 \pm 0.9) \mathrm{nm}$. These values are clearly different from those for other molecular weights. This difference may be due to the entanglement effect, because the critical molecular weight at the entanglement limit is $1.3 \times 10^{4}$ for $a$-PS [35]. In the present measurements no obvious molecular weight dependence was observed within experimental accuracy except for $M_{w}=3.6 \times 10^{3}$. However, it is plausible that the values of $\delta$ and $\xi$ are functions of the molecular weight. More precise measurements will be required to detect such molecular weight dependence.

Thin polymer films with both substrate and upper electrodes of $\mathrm{Al}$ for dielectric measurements were used in the present measurements. Due to the presence of these electrodes, for our thin films there was no real free surface existing in the air-polymer interface. The samples discussed presently were capped supported films, according to the terminology given in Ref. [19]. However, the values of $T_{g}$ observed in the present measurements agree with those obtained by ellipsometry for uncapped films. It has also been reported that there is no obvious differences between the results obtained for uncapped and capped supported films [19]. These experimental results support the conclusion that there is no appreciable difference in $T_{g}$ for capped and uncapped supported thin films; i.e., the upper electrode of our samples can be assumed to have no effect on the thermal properties of the polymer films, in particular the thermal expansivity along the direction normal to the substrate. Recent DSC measurements have revealed the existence of a surface mobile layer of PS spheres dispersed in $\mathrm{Al}_{2} \mathrm{O}_{3}$ powders [36]. According to the discussion given by Mayes [37], the glass transition temperature of a (surface) layer can be depressed only if the end concentration of the layer is higher than that 


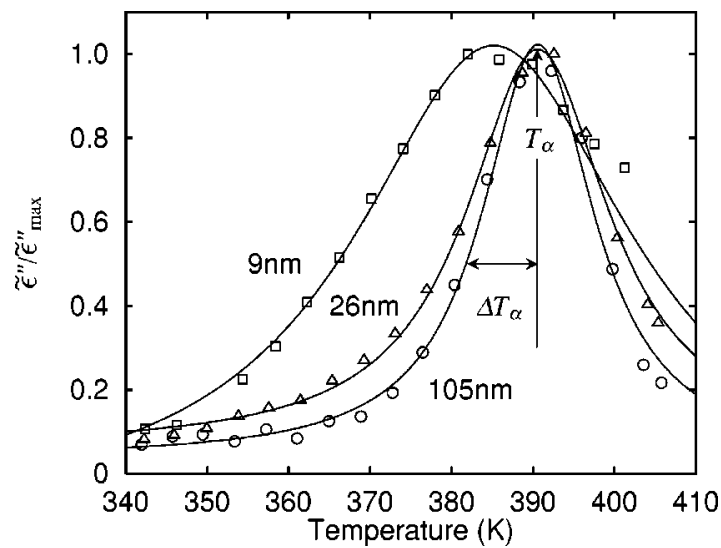

FIG. 4. Reduced dielectric loss as a function of temperature for various film thicknesses $\left(M_{w}=2.8 \times 10^{5}\right.$ and $\left.f=100 \mathrm{~Hz}\right)$. The symbol $\bigcirc$ corresponds to $d=105 \mathrm{~nm}, \triangle$ to $d=26 \mathrm{~nm}$, and $\square$ to $d$ $=9 \mathrm{~nm}$. The curves were obtained by fitting the data to the equation $\tilde{\epsilon}^{\prime \prime}=\widetilde{\epsilon}_{\max }^{\prime \prime} /\left\{1+\left[\left(T-T_{\alpha}\right) / \Delta T_{\alpha}\right]^{2}\right\}$.

of bulk samples. The existence of a true free surface is not a necessary condition for the decrease of $T_{g}$ and the existence of a liquidlike layer.

Recent measurements of the mass density of $a$-PS thin films supported on Si using neutron reflectivity show that the average mass density within films is near the bulk value regardless of film thickness [38]. Sound velocities in thin freely standing PS films measured by Brillouin light scattering are reported to be the same for all films with various film thicknesses. This also suggests that the average mass density of thin films is the same as that of bulk samples [39]. In the presently considered layer model it is assumed that there are thin liquidlike and dead layers in addition to the layer with bulk properties. Because the liquidlike layer has a lower mass density and the dead layer has a higher mass density than the bulk layer, it is not unreasonable to assume that the average mass density of these thin films is the same as that of the bulk samples. In the case of freely standing films, because there is no dead layer, the average mass density is expected to become lower for very thin films than that of bulk sample. However, the observed value of the average mass density does not change with film thickness. The simple layer model may no longer be valid for freely standing films, and it may be the case that another physical factor must be taken into account.

It should also be noted here that picosecond acoustic techniques reveal an increase in the longitudinal sound velocity for thin films of poly(methyl methacrylate) and PS [40,41]. This suggests a change in the average mass density for thin films from that of bulk samples. This result disagrees with that obtained in Refs. [38,39].

\section{DYNAMICS OF THE $\alpha$ PROCESS OF THIN FILMS}

In this section results concerning dielectric loss during the heating process are given to allow for discussion how the dynamics of the $\alpha$-process changes with decreasing $T_{g}$ as a result of decreasing thickness. First, the temperature dependence of the dielectric loss with a fixed frequency is investigated to directly compare the thickness dependence of $T_{g}$ with that of the dynamics of the $\alpha$ process. Second, the re-
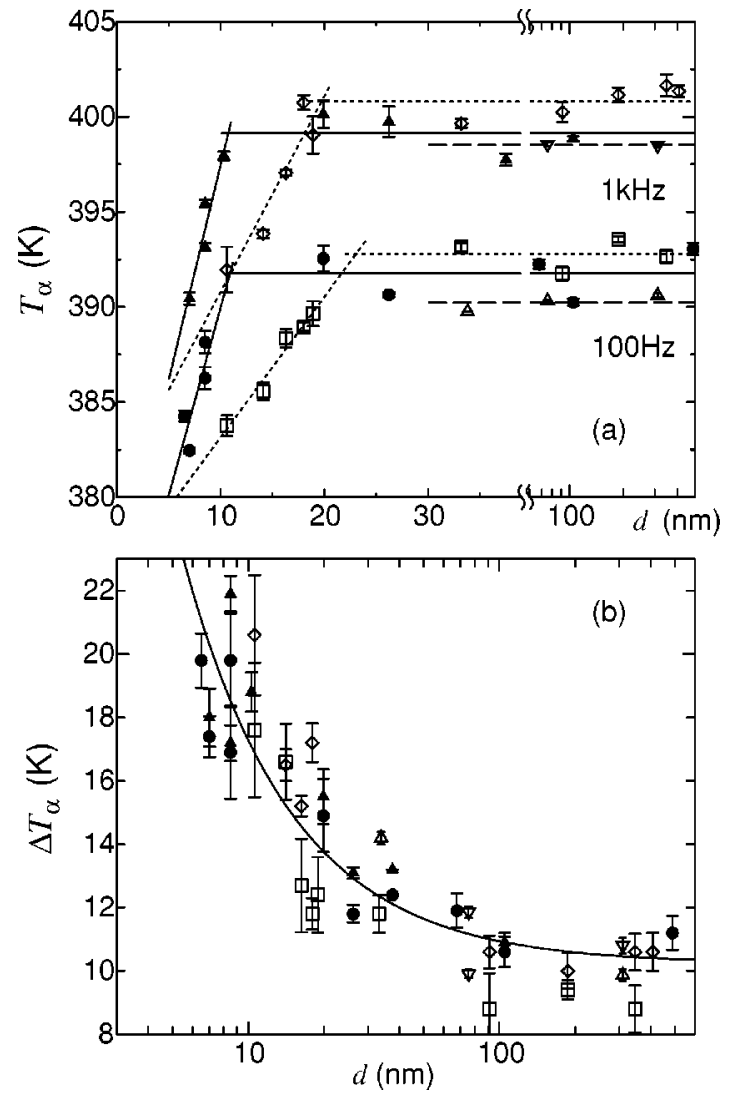

FIG. 5. Thickness dependence of (a) $T_{\alpha}$ and (b) $\Delta T_{\alpha}$ during the heating process for a given frequency. The symbol $\bigcirc$ corresponds to $f=100 \mathrm{~Hz}$ and $M_{w}=2.8 \times 10^{5}, \boldsymbol{\Delta}$ to $f=1 \mathrm{kHz}$ and $M_{w}=$ $2.8 \times 10^{5}, \square$ to $f=100 \mathrm{~Hz}$ and $M_{w}=1.8 \times 10^{6}, \diamond$ to $f=1 \mathrm{kHz}$ and $M_{w}=1.8 \times 10^{6}, \triangle$ to $f=100 \mathrm{~Hz}$ and $M_{w}=3.6 \times 10^{4}$, and $\nabla$ to $f$ $=1 \mathrm{kHz}$ and $M_{w}=3.6 \times 10^{4}$. The solid lines in (a) were drawn for $M_{w}=2.8 \times 10^{5}$, the dotted lines for $M_{w}=1.8 \times 10^{6}$, and the broken lines for $M_{w}=3.6 \times 10^{4}$. These lines were drawn using Eq. (15) and the curve in (b) was obtained from Eq. (14).

sults for the dielectric loss in the frequency domain under an isothermal condition are given to confirm the results obtained with fixed frequency and to allow for discussion of the relaxation behavior due to the $\alpha$ process in thin polymer films.

\section{A. Dielectric loss with fixed frequency}

Figure 4 shows the reduced dielectric loss $\tilde{\epsilon}^{\prime \prime} / \tilde{\epsilon}_{\max }^{\prime \prime}$ as a function of temperature at $100 \mathrm{~Hz}$ in $a$-PS samples of thickness 9,26 , and $105 \mathrm{~nm}$ with $M_{w}=2.8 \times 10^{5}$. Here, the reduced dielectric loss is defined by $\tilde{\epsilon}^{\prime \prime} / \widetilde{\epsilon}_{\max }^{\prime \prime}=\left[C^{\prime \prime}(T)\right.$ $\left.-C^{\prime \prime}\left(T_{0}\right)\right] /\left[C_{\max }^{\prime \prime}\left(T_{\alpha}\right)-C^{\prime \prime}\left(T_{0}\right)\right]$, where $C^{\prime \prime}$ is the imaginary part of the complex capacitance, $T_{0}$ is a standard temperature (in this case, $T_{0}$ is room temperature), and $C_{\max }^{\prime \prime}$ is the peak value of $C^{\prime \prime}(T)$ due to the $\alpha$ process. Above $T_{g}$ the dielectric loss $\tilde{\epsilon}^{\prime \prime} / \tilde{\epsilon}_{\max }^{\prime \prime}$ for a given frequency displays an anomalous increase with temperature due to the $\alpha$ process, and it possesses a maximum at the temperature $T_{\alpha}$. The value of $T_{\alpha}$ and the width of the $\alpha$ peak, $\Delta T_{\alpha}$, also depend on $d$, as shown in Fig. 4. Here, $\Delta T_{\alpha}$ is defined as the temperature difference between $T_{\alpha}$ and the lower temperature at which $\widetilde{\epsilon}^{\prime \prime}$ is half its peak value. As shown in Fig. 5(b), the width $\Delta T_{\alpha}$ 
TABLE II. The values of $T_{\alpha}^{\infty}, d_{c}$, and $\zeta$ resulting in the best fit for thin films of $a$-PS with $M_{w}=2.8 \times 10^{5}$ and $1.8 \times 10^{6}$.

\begin{tabular}{lcccc}
\hline \hline$M_{w}$ & $f(\mathrm{~Hz})$ & $T_{\alpha}^{\infty}(\mathrm{K})$ & $d_{\mathrm{c}}(\mathrm{nm})$ & $\zeta\left(10^{2} \mathrm{~nm}\right)$ \\
\hline $2.8 \times 10^{5}$ & 100 & $391.8 \pm 0.5$ & $10.9 \pm 1.1$ & $2.0 \pm 0.7$ \\
& 1000 & $398.9 \pm 0.4$ & $10.3 \pm 0.7$ & $1.6 \pm 0.5$ \\
$1.8 \times 10^{6}$ & 100 & $392.8 \pm 0.3$ & $23.1 \pm 0.9$ & $5.3 \pm 0.6$ \\
& 1000 & $400.8 \pm 0.4$ & $19.7 \pm 0.8$ & $3.9 \pm 0.5$ \\
\hline \hline
\end{tabular}

begins to increase at about $100 \mathrm{~nm}$ and continues to increase monotonically with decreasing $d$. The $d$ dependence of $\Delta T_{\alpha}$ can be expressed as

$$
\Delta T_{\alpha}(d)=\Delta T_{\alpha}^{\infty}\left(1+\frac{a^{\prime}}{d}\right),
$$

where $a^{\prime}=6.9 \pm 0.8 \mathrm{~nm}$ and $\Delta T_{\alpha}^{\infty}=10.3 \pm 0.4 \mathrm{~K}$. Comparing the $d$ dependence of $\Delta T_{\alpha}$ with that of $T_{g}$ (Fig. 2), we find that the decrease of $T_{g}$ is directly correlated with the broadening of the $\alpha$ peak as $\delta\left(T_{g}(d)\right) / T_{g}^{\infty}=$ $-C_{1} \delta\left(\Delta T_{\alpha}(d)\right) / \Delta T_{\alpha}^{\infty}, \quad$ where $\quad \delta T_{g}(d)=T_{g}(d)-T_{g}^{\infty}$, $\delta\left(\Delta T_{\alpha}(d)\right)=\Delta T_{\alpha}(d)-\Delta T_{\alpha}^{\infty}$, and $C_{1}$ is a constant $(4.8$ $\left.\times 10^{-2}-6.5 \times 10^{-2}\right)$. In other words, it can be concluded that the broadening of the distribution of relaxation times for the $\alpha$ process is closely correlated to the decrease of $T_{g}$.

Contrastingly, Fig. 5(a) shows that $T_{\alpha}$ remains almost constant as $d$ is decreased, down to the critical thickness $d_{c}$, at which point it begins to decrease linearly with decreasing $d$. Therefore, $T_{\alpha}$ is given as follows:

$$
T_{\alpha}(d)= \begin{cases}T_{\alpha}^{\infty}, & d>d_{c} \\ T_{\alpha}^{\infty}\left(1+\frac{d-d_{\mathrm{c}}}{\zeta}\right), & d<d_{c},\end{cases}
$$

where $T_{\alpha}^{\infty}$ and $\zeta$ are constants. The functional form of $T_{\alpha}$ with respect to $d$ is independent of $M_{w}$, because Eq. (15) can well reproduce experimental values of $T_{\alpha}$ for two different molecular weights $M_{w}=2.8 \times 10^{5}$ and $1.8 \times 10^{6}$. The parameters $d_{c}$ and $\zeta$ show a distinct molecular weight dependence as shown in Table II. The $M_{w}$ and $d$ dependences of $T_{\alpha}$ are quite different from those of $T_{g}$ and $\Delta T_{\alpha}$ found in the present and previous measurements on supported PS films $[15,19]$. They are similar to those of $T_{g}$ for freely standing films of $a$-PS [18].

The values of $d_{c}$ listed in Table II are $d_{c}=11 \mathrm{~nm}$ for $M_{w}=2.8 \times 10^{5}$ and $d_{c}=20-23 \mathrm{~nm}$ for $M_{w}=1.8 \times 10^{6}$. These values seem to be related to the radius of gyration of the bulk polymer coil $\left[R_{g}=0.028 \times \sqrt{M}(\mathrm{~nm})[17]\right]: R_{g}=15 \mathrm{~nm}$ for $M_{w}=2.8 \times 10^{5}$ and $38 \mathrm{~nm}$ for $M_{w}=1.8 \times 10^{6}$. Furthermore, if we assume that $d_{c}$ and $\zeta$ can be scaled by the functional form of $d_{c} \sim M^{\epsilon}$ and $\zeta \sim M^{\gamma}$, where $M$ is the molecular weight of polymers, the values of $\epsilon$ and $\gamma$ can be estimated as follows: $\epsilon=0.38 \pm 0.10$ and $\gamma=0.51 \pm 0.08$. These values are nearly equal or similar to the exponent of radius of gyration for Gaussian chains $(\nu=0.5)$. In the molten state, polymer chains can be regarded as Gaussian chains. This result suggests that the length scale such as the radius of gyration of the bulk polymer may control the drastic change of $T_{\alpha}$ near and below $d_{\mathrm{c}}$. In other words, the deformation of random coils of polymer chains confined in thin films may cause the observed decrease in $T_{\alpha}$ with decreasing film thickness. As discussed later, one of the possible origins of this deformation is the competition between the liquidlike layer and the dead layer. The investigation of $d$ dependence of $T_{\alpha}$ for various $M_{w}$ samples will reveal the detailed mechanism of a drastic decrease in $T_{\alpha}$ with decreasing $d$.

Here it should be noted that the length scale $a^{\prime}$ included in the expression of $\Delta T_{\alpha}$ as a function of $1 / d$ is much larger than $a$ in $T_{\mathrm{g}}: a^{\prime} / a=15.3$. This can be explained in the following way. It is assumed that the shape of the loss peak due to the $\alpha$ process in the plot of $\epsilon^{\prime \prime}$ vs $T$ can be expressed by the same function of $T_{\alpha}$ and $\Delta T_{\alpha}$ for any film thickness $d$ and that $T_{g}$ can be regarded as the temperature at which dielectric loss begins to increase due to the $\alpha$ process. From this assumption, the following relation can be obtained:

$$
T_{\alpha}(d)-T_{g}(d)=A \Delta T_{\alpha}(d),
$$

where $A$ is a constant. Taking into account that for $d>d_{c}$, $T_{\alpha}(d)=T_{\alpha}^{\infty}$, and substituting Eqs. (10) and (14) into Eq. (16), we obtain the relations $T_{\alpha}^{\infty}-T_{g}^{\infty}=A \Delta T_{\alpha}^{\infty}$ and $T_{g}^{\infty} a$ $=A \Delta T_{\alpha}^{\infty} a^{\prime}$. Hence, the value of $a^{\prime} / a$ is expressed by

$$
\frac{a^{\prime}}{a}=\frac{T_{g}^{\infty}}{T_{\alpha}^{\infty}-T_{g}^{\infty}} .
$$

Using Eq. (17) with the observed values of $T_{g}^{\infty}$ and $T_{\alpha}^{\infty}$ we obtain $a^{\prime} / a \approx 17$ for $f=100 \mathrm{~Hz}$ and 12 for $f=1 \mathrm{kHz}$. Although the errors in $\Delta T_{\alpha}$ prevent us from obtaining the frequency dependence of $a^{\prime}$, the values of $a^{\prime} / a$ evaluated using the above assumption agree well with those found in the present measurements.

Recently, Forrest et al. obtained $\alpha$ relaxation data with a characteristic time scale $\langle\tau\rangle \sim 2 \times 10^{-4}$ s using a quartz crystal microbalance technique applied to supported PS films covered with $\mathrm{SiC}$ particles [24]. It was reported that the small dissipation peak $T_{\text {max }}$, which corresponds to $T_{\alpha}$ in this paper, exhibits the same $d$ dependence as $T_{g}$ when the values of $T_{g}$ are shifted by $20 \mathrm{~K}$. The $d$ dependence of $T_{\max }$ found from their measurements seems to be different from that found from the present measurements. The values of $T_{g}$ used for comparison with $T_{\max }$ were observed by ellipsometry for PS films supported on a hydrogen-passivated $\mathrm{Si}$ substrate with a free surface [34]. Hence, the comparison between $T_{g}$ and $T_{\max }$ in Ref. [24] was carried out for samples with different molecular weight and of different geometries (with and without a free surface) by using different experimental techniques, while a direct comparison was carried out in our study by using simultaneous measurements of $T_{g}$ and $T_{\alpha}$ for the same sample. The results obtained in Ref. [24] may have been affected by a small difference in experimental conditions for the two different measurements of $T_{g}$ and $T_{\max }$. Furthermore, $T_{\max }$ plotted in the inset of Fig. 2 of Ref. [24] can also be fitted by the equation proposed for $T_{\alpha}$ in this paper [see Eq. (15)], and the critical thickness $d_{\mathrm{c}}$ is found to be $35 \mathrm{~nm}$. 

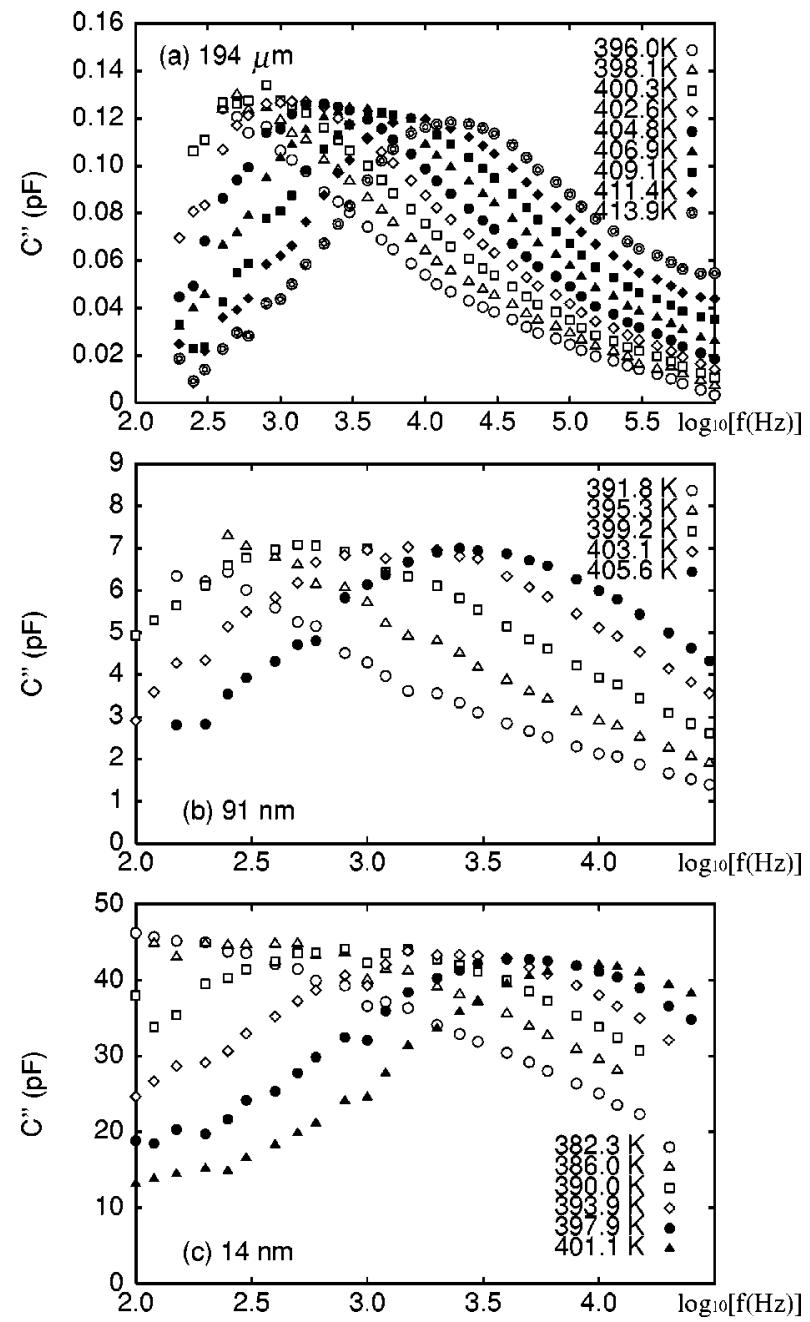

FIG. 6. Frequency dependence of the imaginary part of the complex capacitance for $a$-PS thin films with various film thicknesses $\left(M_{w}=1.8 \times 10^{6}\right)$ : (a) $d=194 \mu \mathrm{m}$ (bulk sample); (b) $d=91 \mathrm{~nm}$; (c) $d=14 \mathrm{~nm}$.

\section{B. Dielectric relaxation behavior of thin films}

Here, we give the results for the imaginary part of the complex capacitance (dielectric loss) as a function of the frequency to facilitate discussion of the dynamics of thin films of $a$-PS with various film thicknesses between $14 \mathrm{~nm}$ and $194 \mu \mathrm{m}$. Figure 6 displays the dielectric loss vs frequency at various temperatures above $T_{g}$ for $a$-PS $\left(M_{w}\right.$ $=1.8 \times 10^{6}$ ) with film thicknesses of (a) $194 \mu \mathrm{m}$ (bulk sample), (b) $91 \mathrm{~nm}$, and (c) $14 \mathrm{~nm}$. The peak in Fig. 6 corresponds to that due to the $\alpha$ process. It it found that the relaxation behavior of the $\alpha$ process changes with temperature and thickness. The peak frequency shifts to the higher frequency side as the temperature increases. In Fig. 7 the peak frequency $f_{\max }$, which corresponds to the inverse of the relaxation time $\tau_{\alpha}$ of the $\alpha$ process, is plotted as a function of the inverse temperature. It is found that the values of $\tau_{\alpha}$ for the films with thickness from $33 \mathrm{~nm}$ to $194 \mu m$ fall on the same curve, which can be described by the VogelFulcher-Tammann (VFT) equation. Here it should be noted that the values of $T_{g}$ for the films with thickness of 33 and 91 $\mathrm{nm}$ are smaller than those of thicker films, although $\tau_{\alpha}$ remains constant. As the thickness decreases further, down to

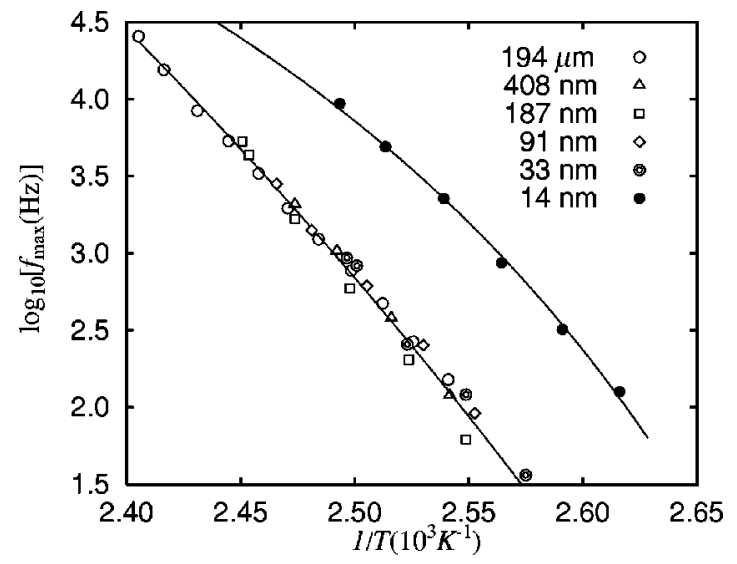

FIG. 7. Peak frequency of dielectric loss due to the $\alpha$ process as a function of the inverse of temperature for thin films of $a$-PS with various film thicknesses $\left(M_{w}=1.8 \times 10^{6}\right)$. Solid curves were obtained by fitting the data to the VFT equation, $f_{\max }=f_{0} \exp$ $\left[-U /\left(T-T_{V}\right)\right]$, where $f_{0}, U$, and $T_{V}$ are constants.

$14 \mathrm{~nm}, \tau_{\alpha}$ becomes much shorter than that for thicker films. It follows from this result that the relaxation time $\tau_{\alpha}$ of the $\alpha$ process remains constant down to a critical thickness, below which it begins to decrease. This result is consistent with that extracted from the experimental observations of dielectric loss with fixed frequency discussed in Sec. IV A.

In order to discuss the relaxation behavior of the $\alpha$ process, the profiles in Fig. 6 are replotted by scaling them with respect to peak positions and peak heights. Figure 8 shows that profiles of dielectric loss vs frequency can be reduced in this way to a single master curve over the temperature range above $T_{g}$ described in the figure captions. It it clearly found that peak profiles become broader as the film thickness decreases. The master curve for each film with different thickness can be fitted by using the Havriliak-Negami (HN) equation [42],

$$
\epsilon^{\prime \prime}(\omega)=\operatorname{Im} \frac{\Delta \epsilon}{\left[1+\left(i \omega \tau_{0}\right)^{\left.1-\alpha_{\mathrm{HN}}\right]^{\beta_{\mathrm{HN}}}}\right.},
$$

where $\Delta \epsilon$ is the dielectric strength, $\omega$ is angular frequency $(=2 \pi f), \tau_{0}$ is the apparent relaxation time, and $\alpha_{\mathrm{HN}}$ and $\beta_{\mathrm{HN}}$ are shape parameters. The values of $\alpha_{\mathrm{HN}}$ and $\beta_{\mathrm{HN}}$ resulting in the best fit are given in Table III. The solid curves given in Fig. 8 are calculated using the HN equation.

Here, the data in the frequency domain are converted into those in time domain. Using the equation

$$
\phi(t)=\frac{2}{\pi} \int_{0}^{\infty} \frac{\epsilon^{\prime \prime}(\omega)}{\Delta \epsilon} \cos \omega t \frac{d \omega}{d t},
$$

the relaxation function $\phi(t)$ can be calculated via the $\mathrm{HN}$ equation with the best-fit parameters for thin films of various thicknesses, as shown in Fig. 9. The shape of the relaxation function changes with film thickness. As seen in Fig. 9, the relaxation function thus obtained can be fitted quite well by the Kahlrausch-Williams-Watts (KWW) equation

$$
\phi(t)=\exp \left[-\left(\frac{t}{\tau_{\mathrm{KWW}}}\right)^{\beta_{\mathrm{KWw}}}\right]
$$




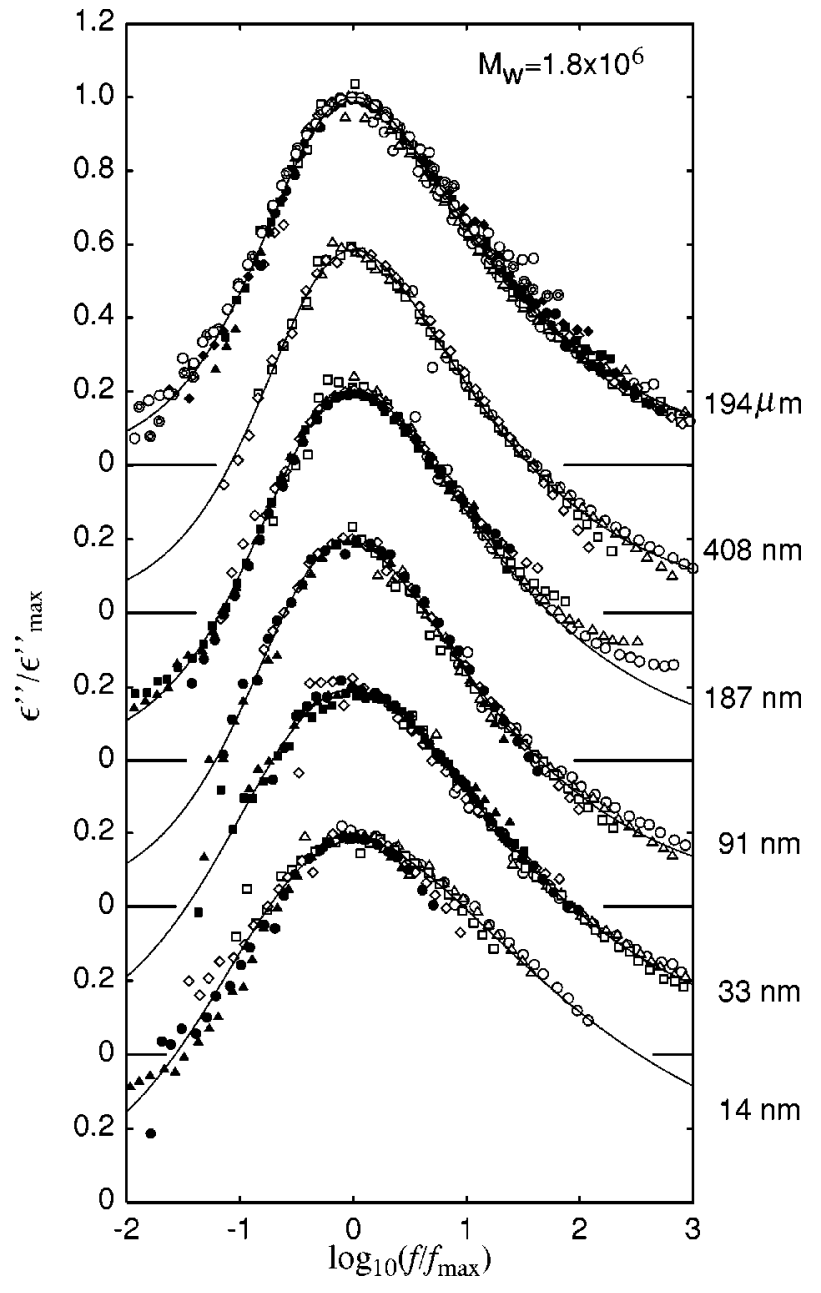

FIG. 8. Dependence of the normalized dielectric loss on the logarithm of the normalized frequency $\left(M_{w}=1.8 \times 10^{6}\right)$. The two axes are normalized with respect to the peak position due to the $\alpha$ process, corresponding to $\epsilon_{\max }^{\prime \prime}$ and $f_{\max }$. The numbers given in the right margin are the film thicknesses. The solid curves for each film with various thicknesses were obtained by fitting the data to the $\mathrm{HN}$ equation. The parameter values resulting in the best fit are given in Table II. For $d=194 \mu \mathrm{m}, 91 \mathrm{~nm}$, and $14 \mathrm{~nm}$, the symbols are the same as in Fig. 6. For $d=408 \mathrm{~nm}$, the symbol $\bigcirc$ corresponds to $393.5 \mathrm{~K}, \triangle$ to $397.5 \mathrm{~K}, \square$ to $401.3 \mathrm{~K}$, and $\diamond$ to $404.3 \mathrm{~K}$. For $d$ $=187 \mathrm{~nm}$, the symbol $\bigcirc$ corresponds to $388.5 \mathrm{~K}, \triangle$ to $392.4 \mathrm{~K}, \square$ to $396.3 \mathrm{~K}, \diamond$ to $400.4 \mathrm{~K}, \bigcirc$ to $404.3 \mathrm{~K}, \boldsymbol{\Delta}$ to $407.6 \mathrm{~K}$, and $\mathbf{\square}$ to $408.1 \mathrm{~K}$. For $d=33 \mathrm{~nm}$, the symbol $\bigcirc$ corresponds to $380.5 \mathrm{~K}, \triangle$ to $384.5 \mathrm{~K}, \square$ to $388.4 \mathrm{~K}, \diamond$ to $392.4 \mathrm{~K}, \diamond$ to $396.4 \mathrm{~K}, \boldsymbol{\Delta}$ to 399.9 $\mathrm{K}$, and $\mathbf{\square}$ to $400.6 \mathrm{~K}$.

TABLE III. The values of $\alpha_{\mathrm{HN}}, \beta_{\mathrm{HN}}$, and $\beta_{\mathrm{KWW}}$ resulting in the best fit for thin films of $a$-PS with $M_{\mathrm{w}}=1.8 \times 10^{6}$.

\begin{tabular}{lccc}
\hline \hline$d(\mathrm{~nm})$ & $\alpha_{\mathrm{HN}}$ & $\beta_{\mathrm{HN}}$ & $\beta_{\mathrm{KWW}}$ \\
\hline $194 \times 10^{3}$ & $0.22 \pm 0.01$ & $0.46 \pm 0.01$ & $0.419 \pm 0.012$ \\
408 & $0.22 \pm 0.01$ & $0.48 \pm 0.02$ & $0.435 \pm 0.017$ \\
187 & $0.25 \pm 0.01$ & $0.46 \pm 0.03$ & $0.399 \pm 0.021$ \\
91 & $0.25 \pm 0.01$ & $0.47 \pm 0.01$ & $0.406 \pm 0.014$ \\
33 & $0.38 \pm 0.01$ & $0.51 \pm 0.02$ & $0.344 \pm 0.017$ \\
14 & $0.40 \pm 0.02$ & $0.37 \pm 0.03$ & $0.271 \pm 0.019$ \\
\hline \hline
\end{tabular}

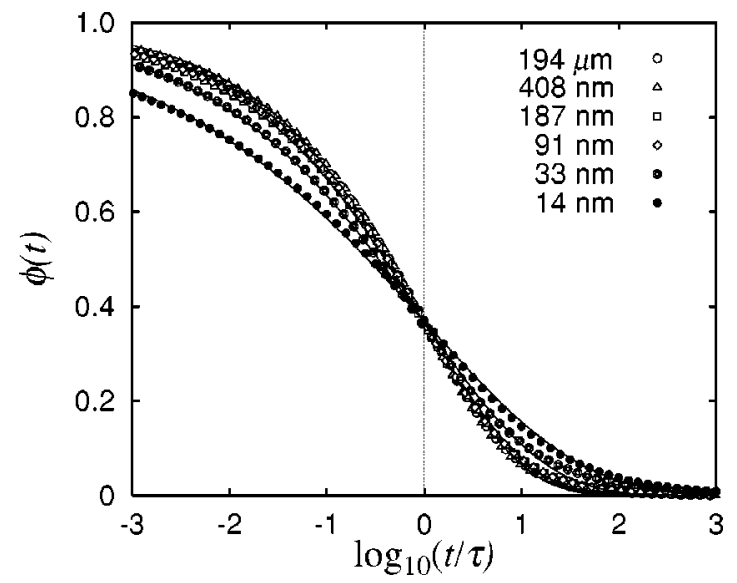

FIG. 9. The relaxation function $\phi$ as a function of the logarithm of the reduced time $t / \tau_{\mathrm{KWW}}$ for $a$-PS thin films with various thicknesses. The relaxation functions plotted by the six different symbols were calculated using Eqs. (18) and (19) with the best-fit parameters $\alpha_{\mathrm{HN}}$ and $\beta_{\mathrm{HN}}$ listed in Table II for each thickness. The solid curves were obtained by fitting the relaxation functions to the KWW equation.

for any thickness. It is also found that the relaxation behavior becomes slower as the film thickness decreases.

Figure 10 displays the exponent $\beta_{\mathrm{KWW}}$ as a function of the inverse of the film thickness. It it found that $\beta_{\mathrm{KWW}}$ decreases from 0.42 to 0.27 as the thickness changes from $194 \mu \mathrm{m}$ to $14 \mathrm{~nm}$. The functional form of $\beta_{\mathrm{KWW}}$ with respect to the inverse of the thickness is found to be linear:

$$
\beta_{\mathrm{KWW}}=\beta_{\mathrm{KWW}}^{\infty}\left(1-\frac{a^{\prime \prime}}{d}\right),
$$

where $\beta_{\mathrm{KWW}}^{\infty}=0.423 \pm 0.006$ and $a^{\prime \prime}=(5.2 \pm 0.4) \mathrm{nm}$. The value of $\beta_{\mathrm{KWW}}$ is a measure of the distribution of relaxation times $\tau_{\alpha}$ of the $\alpha$ process, i.e., the distribution becomes broader as $\beta_{\mathrm{KWW}}$ becomes smaller. Therefore, as the thickness decreases, the distribution of the relaxation times $\tau_{\alpha}$ becomes broader according to Eq. (21). The characteristic length scale $a^{\prime \prime}$ obtained in this analysis of $\beta_{\mathrm{KWW}}$ is consistent with the value $a^{\prime}$ obtained for $\Delta T_{\alpha}$. Furthermore, the

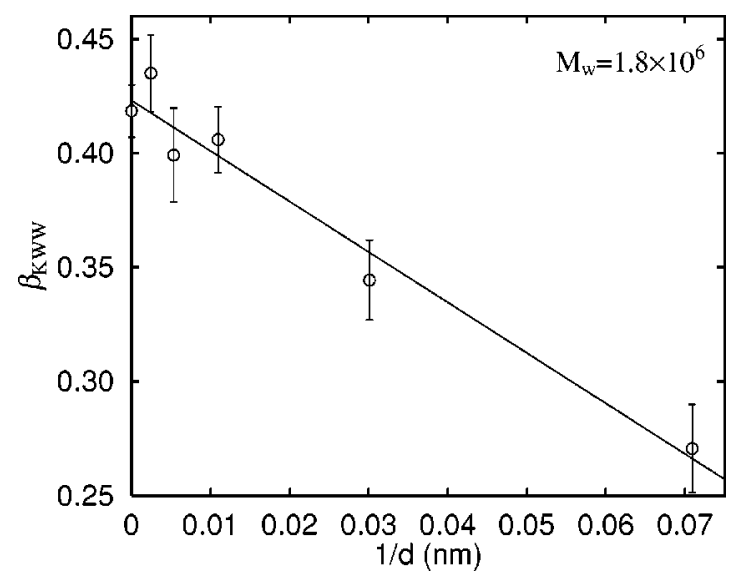

FIG. 10. The KWW exponent $\beta_{\mathrm{KWW}}$ as a function of the inverse of the thickness $d\left(M_{w}=1.8 \times 10^{6}\right)$. The values $\beta_{\mathrm{Kww}}$ were obtained by fitting the relaxation function to the KWW equation. The solid line was plotted using Eq. (21). 
relative change of $\beta_{\mathrm{KWw}}$ measured with respect to that of the bulk sample, $\delta \beta_{\mathrm{KWW}}(d)=\beta_{\mathrm{KWW}}^{\infty}-\beta_{\mathrm{KWW}}(d)$, is directly related to the relative change of $T_{g}$ as follows:

$$
\frac{\delta T_{g}(d)}{T_{g}^{\infty}}=9.6 \times 10^{-2} \times \frac{\delta \beta_{\mathrm{KWW}}(d)}{\beta_{\mathrm{KWW}}^{\infty}} .
$$

The values of $\beta_{\mathrm{KWw}}$ for freely standing films of $a$-PS have been evaluated using photon correlation spectroscopy and found to be indistinguishable from those of bulk PS [24]. On the other hand, a decrease in $\beta_{\mathrm{KWW}}$ has been observed for a copolymer thin film supported on quartz [23], as found in the present measurements on PS supported on a glass substrate. We thus conclude that there is a large difference in the dynamics of freely standing films and supported thin films. In the case of thin films supported on substrate, it is easily understood that the existence of the substrate may cause a broadening of the distribution of $\alpha$-relaxation times, because the dynamics of polymer chains should depend on the distance from the substrate near the boundary.

Although only the results for the dielectric loss in thin films of $a$-PS with $M_{w}=1.8 \times 10^{6}$ are given in Sec. IV B, the results obtained for thin films with $M_{w}=2.8 \times 10^{5}$ are consistent with these.

\section{DISCUSSION AND SUMMARY}

In the case of thin polymer films supported on substrate, not only the surface effects but also the interactions between the substrate and films strongly affect the dynamics and the glass transition of the thin films. We introduced a three-layer model in order to explain such surface and interfacial effects in Sec. III B, following Ref. [17]. In this model it is assumed that within a thin film there are a liquidlike layer and a dead layer in addition to a bulklike layer.

According to this model, two different $\alpha$ processes should exist corresponding to the liquidlike layer and bulklike layer. (If the $\alpha$ process exists in the dead layer, it should exist only far above $T_{g}$ of the bulk sample, i.e., beyond the experimentally accessible temperature range.) We now are performing dielectric measurements to investigate the dynamical properties of $a$-PS thin films over a wider temperature range. Although the measurements are still in progress, direct evidence for the existence of different processes in thin films of $a$-PS is displayed in Fig. 11. Figure 11 shows the normalized dielectric loss as a function of temperature for $a$-PS thin films with thicknesses of 105 and $9 \mathrm{~nm}$ and $M_{w}=2.8 \times 10^{5}$. In this case, the standard temperature $T_{0}$ is set to $180 \mathrm{~K}$. For films with $d=105$ and $9 \mathrm{~nm}$ there the $\alpha$ process exists around $390 \mathrm{~K}$ for $f=100 \mathrm{~Hz}$, as discussed in Sec. IV, while for films with $d=9 \mathrm{~nm}$ there another $\alpha$ process exists at lower temperature. We refer to the latter as the $\alpha_{1}$ process (see arrows in the figure). This peak in the dielectric loss due to the $\alpha_{1}$ process shifts to the higher temperature side as the frequency of the applied electric field changes from $100 \mathrm{~Hz}$ to $1 \mathrm{kHz}$. This suggests that the dynamical behavior of the peak is similar to that of the $\alpha$ process. Since within the model the thickness of the liquidlike layer is assumed to be constant (independent of the thickness $d$ ) the contribution of the liquidlike layer should become more appreciable as the
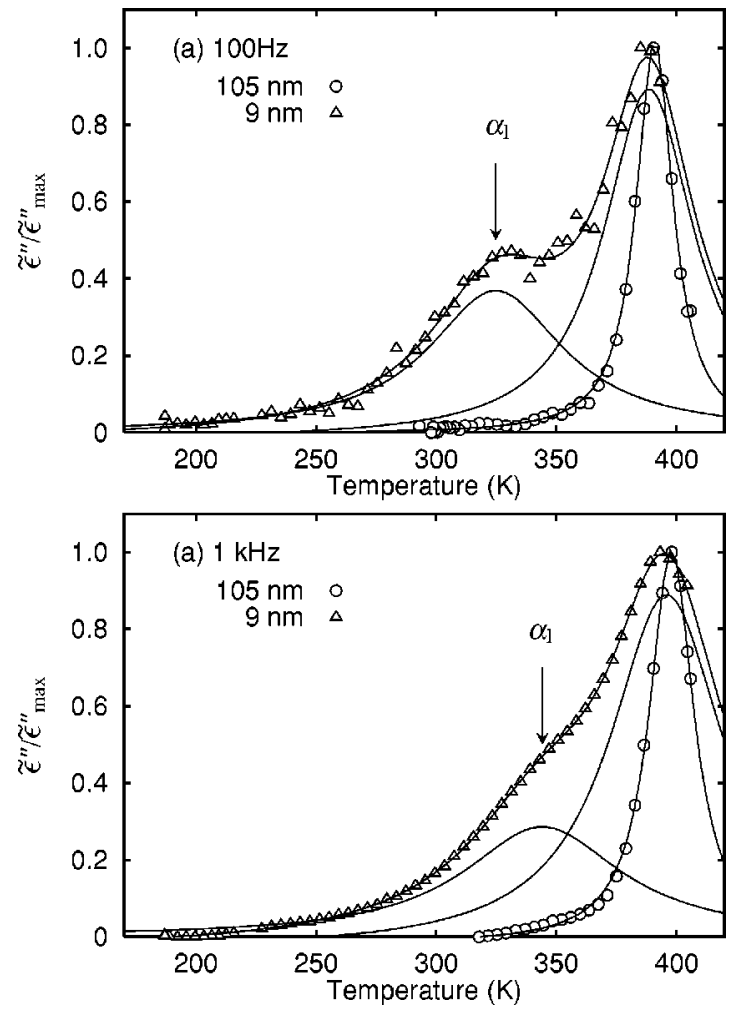

FIG. 11. Reduced dielectric loss as a function of temperature during the cooling process for $a$-PS with film thicknesses of 105 and $9 \mathrm{~nm}\left(M_{w}=2.8 \times 10^{5}\right)$ : (a) $f=100 \mathrm{~Hz}$ and (b) $f=1 \mathrm{kHz}$. The solid curves for the $\alpha_{1}$ process were obtained by fitting the data to the equation $\widetilde{\epsilon}^{\prime \prime}=\sum_{j=\alpha, \alpha} \widetilde{\epsilon}_{\max , j}^{\prime \prime} /\left\{1+\left[\left(T-T_{j}\right) / \Delta T_{j}\right]^{2}\right\}$.

thickness decreases. Therefore, it is reasonable to attribute the loss peak for the $\alpha_{1}$ process to the liquidlike layer. In other words, the existence of the $\alpha_{1}$ process can be regarded as experimental evidence of the existence of a liquidlike layer within a thin film.

Since the $\alpha$ process and the $\alpha_{1}$ process can be attributed to the segmental motion of polymer chains in the bulklike layer and liquidlike layer (surface layer), respectively, we can expect that the characteristic time of the $\alpha_{1}$ process is smaller than that of the $\alpha$ process at a given temperature, because polymer chains involved in the $\alpha_{1}$ process has a higher mobility than those in the $\alpha$ process of a bulklike layer. At higher temperatures the segmental motions of the surface layer is expected to become less different from those of the bulklike layer. Therefore, $T_{\alpha_{l}}$ should be smaller than $T_{\alpha}$ at a given frequency and the difference between $T_{\alpha}$ and $T_{\alpha_{1}}$ should become smaller with increasing frequency. Here, $T_{\alpha_{1}}$ is the temperature at which the dielectric loss possesses a maximum due to the $\alpha_{1}$ process at a given frequency. Because the chain end concentration in the surface layer is higher than that in the bulklike layer, $T_{\alpha_{1}}$ can be expected to have a stronger molecular-weight dependence than $T_{\alpha}$ does.

If there are liquidlike and dead layers in addition to a bulklike layer, it is plausible that there are boundary layers between the bulklike layer and the dead layer and between the bulklike layer and the liquidlike layer. The existence of such boundary layers causes the broadening of the distribution of the $\alpha$ relaxation time of the bulklike layer, and this 
broadening is enhanced as the thickness decreases. Because $T_{g}$ can be regarded as the temperature at which the anomalous increase in $\epsilon^{\prime \prime}$ begins, $T_{g}$ decreases as the distribution of $\tau_{\alpha}$ broadens, i.e., $\Delta T_{\alpha}$ increases or $\beta_{\mathrm{KWW}}$ decreases.

Since the liquidlike and dead layers cause the distribution of $\tau_{\alpha}$ to broaden toward the shorter time side and the longer time side, respectively, the relaxation time $\tau_{\alpha}$ can remain constant as long as the two contributions cancel each other. In the present measurements, we measured the peak frequencies $f_{\max }$ in the dielectric loss due to the $\alpha$ process, which are equal to the inverse of $2 \pi \tau_{\alpha}$, and it is found that $f_{\max }$ at a given temperature is almost independent of the thickness down to the critical thickness $d_{c}$.

Below the critical thickness, there is no $\alpha$ process with dynamical properties observed in the bulk sample. In this case the dynamical properties of the thin film are determined by the competition between the liquidlike layer and the dead layer within the thin film. The values of $\tau_{\alpha}$ can no longer remain constant, but it decreases or increases, depending on whether contributions from the liquidlike layer are stronger or weaker than those from the dead layer. In the present case, $\tau_{\alpha}$ and $T_{\alpha}$ decrease drastically for $d<d_{c}$. This behavior can be accounted for by assuming that contributions from the liquidlike layer are much stronger than those from the dead layer.

Here, it should be noted that the observed value of $d_{c}$ is larger than the sum of thickness of the liquidlike layer and the dead layer, $\xi+\delta: d_{c}=11-23 \mathrm{~nm}$, while $\xi+\delta \approx 10 \mathrm{~nm}$. From this result it follows that for $d=d_{c}$, the bulklike layer still exists, but the relaxation time for the $\alpha$ process of the bulklike layer is different from that of the bulk sample. This suggests the possibility that at $d_{\mathrm{c}}$ the thickness of the bulklike layer $(d-\xi-\delta)$ is comparable to the characteristic length scale $\xi_{\mathrm{CRR}}$ of the $\alpha$ process. Therefore, the value of $\xi_{\text {CRR }}$ can be estimated as $1-13 \mathrm{~nm}$, if there is assumed to exist any characteristic length scale of the $\alpha$ process at all. Because $d_{c}$ depends on the molecular weight of $a$-PS, $\xi_{\mathrm{CRR}}$ may also depend on the molecular weight. However, more precise measurements of $\delta$ and $\xi$ showing whether these values depend on the molecular weight are necessary to elucidate the molecular weight dependence of $\xi_{\mathrm{CRR}}$.

In this paper four different length scales, $a, a^{\prime}, a^{\prime \prime}$, and $d_{c}$, were extracted from the dielectric measurements. From the above discussion, $d_{\mathrm{c}}$ is expected to be related to the char- acteristic length scale $\xi_{\mathrm{CRR}}$ for the $\alpha$ process of bulk samples, while the values of $a, a^{\prime}$, and $a^{\prime \prime}$ are believed to be related to the heterogeneous structure of the mobility within thin films or surface and interfacial effects. According to the model of Adam and Gibbs, the size of the CRR increases as the temperature approaches $T_{g}$. Near the glass transition temperature, the characteristic time of the $\alpha$ process is larger than $10^{3} \mathrm{sec}$, and only the slow modes contribute appreciably to the dielectric loss. Hence, a more prominent $d$ dependence of the $\alpha$ process can be expected when the dielectric measurements are performed for frequencies much smaller than those adopted in the present work. We plan to make such measurements in the future.

In this paper we made dielectric measurement on capped thin films of atactic polystyrene supported on an Aldeposited glass substrate. The results can be summarized as follows:

(1) The glass transition temperature of thin films of $a$-PS has been determined using the temperature change in the electric capacitance. The observed $T_{g}$ is consistent with the results obtained by other methods. A decrease in $T_{g}$ with decreasing thickness has been confirmed.

(2) The thermal expansion coefficient normal to the film surface $\alpha_{n}$ increases with decreasing thickness below the apparent $T_{g}$, while it decreases with decreasing thickness above $T_{g}$. The thickness dependence of $\alpha_{n}$ can be described by a linear function of the inverse of the thickness.

(3) The $d$ dependence of $T_{g}$ is directly correlated to that of the width of the peak due to the $\alpha$ process in the temperature domain and also to the distribution of relaxation times of the $\alpha$ process.

(4) The temperature at which dielectric loss is maximal due to the $\alpha$ process in the temperature domain and the $\alpha$ relaxation time obtained by the frequency dependence of the dielectric loss remain constant down to the critical thickness $d_{c}$, while below $d_{c}$ they decrease drastically with decreasing thickness. The values of $d_{c}$ have a molecular weight dependence and are related to the radius of gyration of polymer chains.

\section{ACKNOWLEDGMENTS}

This work was partly supported by a Grant-in-Aid from the Ministry of Education, Science, Sports and Culture of Japan.
[1] Proceedings of the 3rd International Discussion Meeting on Relaxations in Complex Systems [J. Non-Cryst. Solids. 235237, 1 (1998)].

[2] Proceeding of Second Workshop on Non-equilibrium Phenomena in Supercooled Fluids, Glasses and Amorphous Materials, [J. Phys. Condens. Matter 11, 1 (1999)].

[3] M.D. Ediger, C.A. Angell, and S.R. Nagel, J. Phys. Chem. 100, 13201 (1996), and the references cited therein.

[4] G. Adam and J.H. Gibbs, J. Chem. Phys. 43, 139 (1965).

[5] T. Muranaka and Y. Hiwatari, Phys. Rev. E 51, R2735 (1995).

[6] D.N. Perera and P. Harrowell, Phys. Rev. E 54, 1652 (1996).

[7] R. Yamamoto and A. Onuki, J. Phys. Soc. Jpn. 66, 2545 (1997), Phys. Rev. E 58, 3515 (1998).
[8] W. Kob, C. Donan, S.J. Plimton, P.H. Poole, and S.C. Glotzer, Phys. Rev. Lett. 79, 2827 (1997).

[9] K. Schmidt-Rohr and H.W. Spiess, Phys. Rev. Lett. 66, 3020 (1991).

[10] B. Schiener, R. Böhmer, A. Loidl, and R.V. Chamberlin, Science 274, 752 (1996).

[11] M.T. Cicerone and M.D. Ediger, J. Chem. Phys. 103, 5684 (1995).

[12] J. Schüller, Yu. B. Mel'nichenko, R. Richert, and E.W. Fischer, Phys. Rev. Lett. 73, 2224 (1994).

[13] M. Arndt, R. Stannarius, H. Groothues, E. Hempel, and F. Kremer, Phys. Rev. Lett. 79, 2077 (1997).

[14] G. Barut, P. Pissis, R. Pelster, and G. Nimtz, Phys. Rev. Lett. 
80, 3543 (1998); R. Pelster, Phys. Rev. B 59, 9214 (1999).

[15] J.L. Keddie, R.A.L. Jones, and R.A. Cory, Europhys. Lett. 27, 59 (1994).

[16] J.L. Keddie and R.A.L. Jones, Faraday Discuss. 98, 219 (1994).

[17] G.B. DeMaggio, W.E. Frieze, D.W. Gidley, Ming Zhu, H.A. Hristov, and A.F. Yee, Phys. Rev. Lett. 78, 1524 (1997).

[18] J.A. Forrest, K. Dalnoki-Veress, J.R. Stevens, and J.R. Dutcher, Phys. Rev. Lett. 77, 2002 (1996).

[19] J.A. Forrest, K. Dalnoki-Veress, and J.R. Dutcher, Phys. Rev. E 56, 5705 (1997).

[20] W.E. Wallace, J.H. van Zanten, and W.L. Wu, Phys. Rev. E 52, R3329 (1995).

[21] B. Jerome and J. Commandeur, Nature (London) 386, 589 (1997)

[22] B. Jerome, J. Phys.: Condens. Matter 11, A189 (1999).

[23] D.B. Hall, J.C. Hooker, and J.M. Torkelson, Macromolecules 30, 667 (1997).

[24] J.A. Forrest, C. Svanberg, K. Revesz, M. Rodahl, L.M. Torell, and B. Kasemo, Phys. Rev. E 58, R1226 (1998).

[25] T. Kajiyama, K. Tanaka, and A. Takahara, Polymer 39, 4665 (1998).

[26] K. Fukao and Y. Miyamoto, Europhys. Lett. 46, 649 (1999).

[27] C. Bauer, R. Böhmer, S. Moreno-Flores, R. Richert, H. Sillescu, and D. Neher, Phys. Rev. E 61, 1755 (2000).
[28] C. Bauer, R. Richert, and R. Böhmer, J. Non-Cryst. Solids (to be published).

[29] G. Blum, F. Kremer, T. Jaworek, and G. Wegner, Adv. Mater. 7, 1017 (1995).

[30] O. Yano and Y. Wada, J. Polym. Sci., Part A-2 9, 669 (1971).

[31] Polymer Handbook, 3rd ed., edited by J. Brandrup and E.H. Immergut (John Wiley, New York, 1989).

[32] D.G.H. Ballard, G.D. Wignall, and J. Schelten, Eur. Polym. J. 9, 965 (1973).

[33] T.G. Fox, P.J. Flory, J. Appl. Phys. 21, 581 (1950).

[34] J.L. Keddie, R.A.L. Jones, Isr. J. Chem. 35, 21 (1995).

[35] Physical Properties of Polymers Handbook, edited by J.E. Mark (AIP Press, New York, 1996), p. 337.

[36] Zhang Xiaomin, S. Tasaka, M. Kondo, and N. Inagaki, Polym. Prep. Jpn. 48, E470 (1999).

[37] A.M. Mayes, Macromolecules 27, 3114 (1994).

[38] W.E. Wallace, N.C. Beck Tan, W.L. Wu, and S. Satija, J. Chem. Phys. 108, 3798 (1998).

[39] J.A. Forrest, K. Dalnoki-Veress, and J.R. Dutcher, Phys. Rev. E 58, 6109 (1998).

[40] Y.-C. Lee, K.C. Bretz, F.W. Wise, and W. Sachse, Appl. Phys. Lett. 69, 1692 (1996).

[41] K.C. Bretz, Y.-C. Lee, F.W. Wise, and W. Sachse, Bull. Am. Phys. Soc. 42, 648 (1997).

[42] S. Havriliak and S. Negami, Polymer 8, 161 (1967). 